\title{
Correntropy-Based Spectral Characterization of Respiratory Patterns in Patients With Chronic Heart Failure
}

\author{
Ainara Garde, Student Member, IEEE, Leif Sörnmo, Senior Member, IEEE, \\ Raimon Jané, Member, IEEE, and Beatriz F. Giraldo*, Member, IEEE
}

\begin{abstract}
A correntropy-based technique is proposed for the characterization and classification of respiratory flow signals in chronic heart failure (CHF) patients with periodic or nonperiodic breathing (PB or $\mathrm{nPB}$, respectively) and healthy subjects. The correntropy is a recently introduced, generalized correlation measure whose properties lend themselves to the definition of a correntropybased spectral density (CSD). Using this technique, both respiratory and modulation frequencies can be reliably detected at their original positions in the spectrum without prior demodulation of the flow signal. Single-parameter classification of respiratory patterns is investigated for three different parameters extracted from the respiratory and modulation frequency bands of the CSD, and one parameter defined by the correntropy mean. The results show that the ratio between the powers in the modulation and respiratory frequency bands provides the best result when classifying CHF patients with either PB or nPB, yielding an accuracy of $88.9 \%$. The correntropy mean offers excellent performance when classifying CHF patients versus healthy subjects, yielding an accuracy of 95.2\% and discriminating $\mathrm{nPB}$ patients from healthy subjects with an accuracy of $94.4 \%$.
\end{abstract}

Index Terms-Autoregressive (AR) modeling, chronic heart failure (CHF), correntropy spectral density (CSD), linear classification, periodic breathing (PB).

\section{INTRODUCTION}

$\mathbf{C}$ HRONIC HEART FAILURE (CHF) is a serious health problem with a prevalence of $2 \%$ of the U.S. population,

Manuscript received July 28, 2009; revised December 4, 2009 and January 22, 2010; accepted January 22, 2010. Date of publication March 4, 2010; date of current version July 14, 2010. This work was supported in part by the Ministerio de Ciencia e Innovación of the Spanish Government under Grant TEC2007-68076C02-01 and Grant TEC2007-63637. Asterisk indicates corresponding author.

A. Garde and R. Jané are with the Departament d'Enginyeria de Sistemes, Automàtica i Informàtica Industrial, Universitat Politècnica de Catalunya, Barcelona 08028, Spain, and with the Institut de Bioenginyeria de Catalunya, Barcelona 08028, Spain, and also with the Centro de Investigación Biomédica en Red de Bioingeniería, Biomateriales y Nanomedicina, Barcelona 08028, Spain (e-mail: ainara.garde@upc.edu; raimon.jane@upc.edu).

L. Sörnmo is with the Department of Electrical and Information Technology and the Center of Integrative Electrocardiology, Lund University, Lund SE-221 00, Sweden (e-mail: leif.sornmo@eit.lth.se).

*B. F. Giraldo is with the Departament d'Enginyeria de Sistemes, Automàtica i Informàtica Industrial, Escola Universitària d'Enginyeria Técnica Industrial de Barcelona, Universitat Politècnica de Catalunya, Barcelona 08028, Spain, and with the Institut de Bioenginyeria de Catalunya, Barcelona 08028, Spain, and also with the Centro de Investigación Biomédica en Red de Bioingenierìa, Biomateriales y Nanomedicina, Barcelona 08028, Spain (e-mail: beatriz.giraldo@upc.edu).

Color versions of one or more of the figures in this paper are available online at http://ieeexplore.ieee.org.

Digital Object Identifier 10.1109/TBME.2010.2044176 almost 5 million people are affected, and $30 \%$ to $40 \%$ of all patients die within one year after diagnosis [1], [2]. The CHF is associated with major abnormalities of autonomic cardiovascular control, and this group of patients often develop breathing anomalies such as various forms of oscillatory breathing patterns characterized by rises and falls in ventilation. Periodic breathing $(\mathrm{PB})$ patterns can be classified into ventilation with apnea, known as Cheyne-Stokes respiration (CSR), and ventilation without apnea [3], [4]. PB has a prevalence as high as $70 \%$ in CHF patients [5], and is associated with increased mortality [6], especially in CSR patients [7], [8]. The breathing patterns are also influenced by wakefulness or sleep, posture, as well as physiological and mental activity [9]. Physiological parameters for the characterization and detection of different breathing patterns have been suggested in a number of recent clinical studies [10], [11].

Certain studies relate $\mathrm{CHF}$ to alterations in the breathing pattern [12]. For example, the sleep-disordered breathing parameter is associated with an accelerated decline in cardiac function and increased morbidity and mortality [13] in up to $50 \%$ of patients with CHF [14]. Several studies suggest that central sleep apnea is highly prevalent among patients with CHF, and the treatment of this sleep-disordered breathing by continuous positive airway pressure could be an important nonpharmacologic complement to conventional drug therapy [15], [16]. In patients with mild to severe CHF, the power of cardiovascular oscillations at the very low frequency band has been reported to be considerably increased by the presence of $\mathrm{PB}$, which may distort prognosis [17].

Evidence that links exercise capacity to outcome in CHF patients has led to the use of peak oxygen consumption, derived from maximal cardiopulmonary exercise testing, and the exertional oscillatory ventilation index in prognostic assessment. However, these two indexes have certain limitations because of the heterogeneity and complexity of the syndrome [18].

In order to develop new quantitative parameters, our initial studies were focused on the periodicity of the respiratory pattern through the modulation of the respiratory flow signal. The relevant frequency band determined by the frequency peak of the power spectral density (PSD) related to the envelope of the respiratory flow signal was characterized [19], [20]. However, not only is the respiratory modulation frequency essential when studying PB and nPB patterns, respectively, but so is the respiratory frequency.

The correlation function is probably the most widely utilized function in signal processing for quantifying the similarity of 
two random variables. However, as it only considers secondorder statistics, the success of this measure depends on the assumption of Gaussian random variables. Santamaria et al. recently introduced a generalization of the correlation function for stochastic processes, which was called correntropy [21], [22]. Correntropy is a similarity measure defined in terms of inner products of vectors in a kernel parameter space. It involves a positive definite kernel function, which implicitly transforms the original signal into a high-dimensional space in a nonlinear way and calculates efficiently the generalized correlation in that space. This measure, being directly related to Renyi's quadratic entropy estimate of data using Parzen windowing, contains information on both the time structure and the statistical distribution of the underlying dataset, consisting of the information of higher order moments of the data. The ability to reflect nonlinear characteristics of the signal makes correntropy a well-qualified candidate for characterization of nonlinear dynamics. Another attractive property of the correntropy function is its robustness against impulsive noise. Besides, the use of kernel methods makes the correntropy computationally efficient since it can be computed directly from the data.

The aim of the present paper is to study the respiratory flow signal in $\mathrm{CHF}$ patients with $\mathrm{PB}$ and $\mathrm{nPB}$ patterns in order to define parameters, which can improve prognosis and serve as indicators of a patient's condition. The correntropy involves information on higher order statistics, which can be expected to facilitate the detection of respiratory nonlinearities that conventional techniques, based on second-order statistics, are otherwise unable to do.

This paper is organized as follows. Section II briefly describes the correntropy function and the related parametric version of the correntropy-based spectral density (CSD); also, the set of parameters which characterizes the flow signals is described. Section III presents the datasets investigated for performance evaluation, constituted by both real and simulated signals. The classification results are presented in Section III and then discussed in Section IV.

\section{METHODS}

\section{A. Correntropy: Definition and Estimation}

For a discrete-time, strictly stationary stochastic process $x(n), n=0, \ldots, N-1, N$ being the number of samples, the correntropy function $V(m)$ is defined as [21], [23]

$$
V(m)=E[\kappa(x(n), x(n-m))]
$$

where $E[\cdot]$ is the statistical expectation operator and $\kappa(\cdot)$ is a symmetric, positive definite kernel function. In this study, the centered correntropy function $V_{c}(m)$ is employed in which the mean of the transformed data is subtracted so as to reduce the effect of output dc bias [23], [24]. Its definition is given by

$$
\begin{aligned}
V_{c}(m)= & E_{x(n) x(n-m)}[\kappa(x(n), x(n-m))] \\
& -E_{x(n)} E_{x(n-m)}[\kappa(x(n), x(n-m))]
\end{aligned}
$$

where $E_{x(n)} E_{x(n-m)}[\kappa(x(n), x(n-m)]$ is the square mean of the transformed data, corresponding to the correntropy mean value $(\bar{V})$, which can be estimated through the kernel function from the original data

$$
\bar{V}=\frac{1}{N^{2}} \sum_{n=1}^{N} \sum_{n=1}^{N} \kappa(x(n), x(n-m)) .
$$

In the literature, the sigmoidal, Gaussian, polynomial, and spline kernels are among the most commonly used symmetric positive definite kernel functions. They are found in diverse areas such as machine learning, function approximation, density estimation, and support vector machine classification [25], [26]. The Gaussian kernel function, applied in the present study, is given by

$$
\kappa(x(n), x(n-m))=\frac{1}{\sqrt{2 \pi} \sigma} \exp \left[-\frac{(x(n)-x(n-m))^{2}}{2 \sigma^{2}}\right]
$$

where $\sigma$ is referred to as the kernel parameter "standard deviation." With the Taylor series expansion of the Gaussian kernel, we can rewrite the correntropy function as

$$
V(m)=\frac{1}{\sqrt{2 \pi} \sigma} \sum_{k=0}^{\infty} \frac{(-1)^{k}}{2 \sigma^{2 k} k !} E\left[(x(n)-x(n-m))^{2 k}\right]
$$

containing all even-order moments of the random variable $(x(n)-x(n-m))$.

Different kernel functions would yield different expansions, but all kernel functions mentioned earlier are nonlinear and therefore include higher order statistical information about the stochastic process. From (5), it is obvious that the emphasis given to higher order moments is controlled by $\sigma$. In this study, $\sigma$ is determined by Silverman's rule of density estimation [21]:

$$
\sigma=0.9 A N^{-1 / 5}
$$

where $A$ is the smaller value of the standard deviation of data samples and the data interquartile range scaled by 1.34 , and $N$ is the number of samples. Since the flow signals are scalar, this rule is simple to apply and is known to produce reasonable values.

The flow signals sometimes contain missing samples or become saturated, and consequently such samples must be discarded from subsequent analysis as they do not reflect respiratory activity. The samples to be discarded are easily detected however, and are here represented by the binary function $g(n)$, defined by

$$
g(n)= \begin{cases}1 & \text { existing } \\ 0 & \text { discarded }\end{cases}
$$

Information on discarded samples is introduced in the correntropy estimator in a way similar to the sample estimator of the correlation function [27]. Hence, the sample estimator of $V(m)$ in (1) is given by

$$
\hat{V}(m)=\frac{1}{U(m)} \sum_{n=m}^{N} \kappa(x(n), x(n-m)) g(n) g(n-m)
$$

where

$$
U(m)=\sum_{n=m}^{N} g(n) g(n-m)
$$


The results of this study are therefore based on the centered correntropy, estimated by $\hat{V}_{c}(m)=\hat{V}(m)-\bar{V}$.

In addition to the aforementioned signal problems, a variety of other artifacts can be observed in flow signals. In general, these problems have called for various preprocessing algorithms that condition the acquired signal. A powerful advantage of the correntropy is its robustness against impulsive noise, which is due to that the Gaussian kernel makes $\kappa(x(n), x(n-m)) \approx 0$ when either $x(n)$ or $x(n-m)$ is an outlier. Because of this attractive property, the results of the present study were obtained without any kind of preprocessing.

\section{B. Correntropy Spectral Density}

Similar to the conventional correlation function, it can be shown that the correntropy function is positive definite and therefore lends itself for use in many signal processing techniques. Since we are particularly interested in exploring the spectral properties of respiratory flow signals, the CSD is introduced, being a generalization of the conventional PSD [21]. The CSD is based on the Fourier transform of the correntropy function, i.e.

$$
\hat{P}_{x}\left(e^{j \omega}\right)=\sum_{m=-(N-1)}^{N-1} \hat{V}_{c}(m) e^{-j \omega m} .
$$

Considering that the flow signal is oscillatory in nature with a spectrum that exhibits pronounced peaks, autoregressive (AR) spectral analysis is here applied as it offers better spectral resolution than does periodogram-based spectral analysis. The correntropy extension to a parametric CSD, assuming that the signal is characterized by an AR model, is straightforward because the correlation properties are preserved.

In this study, it is essential to have high spectral resolution in the vicinity of the respiratory and modulation peaks; and therefore, the following procedure was adopted. First, the optimum order was determined in each subject using the minimum description length criterion [28]. Then, the maximum of the resulting model order estimates was selected and used as global model order in the subsequent signal analysis. This model order ensures a correct representation of the data when the prediction error is a white sequence. We apply an statistical test (LjungBox) to prove the whiteness of the prediction error.

The AR coefficients can be estimated from the correntropy function, using the Yule-Walker equations, applied here, or any other of the many well-known estimation techniques [29]. This can be done because of the fact that the correntropy matrix, whose elements are defined by $\hat{V}_{c}(m)$, is Toeplitz and positive definite, just as the conventional correlation matrix [21].

\section{Parameter Extraction}

In the time domain, the respiratory pattern is characterized by the correntropy mean, i.e., Renyi's entropy. In the frequency domain, three parameters are derived from two different CSD frequency bands centered either around the respiratory frequency ( $\Delta f_{r}$ : the respiratory frequency bandwidth) or the modulation frequency ( $\Delta f_{m}$ : the modulation frequency bandwidth). The
TABLE I

PARAMETER DESCRIPTION

\begin{tabular}{cl}
\hline Parameter & Description \\
\hline$P_{m}$ & Power of the modulation frequency band \\
$P_{r}$ & Power of the respiratory frequency band \\
$R$ & $P_{m} / P_{r}$ \\
$\bar{V}$ & Correntropy mean \\
\hline
\end{tabular}

modulation frequency of $\mathrm{PB}$ patterns ranges typically from 0.005 to $0.05 \mathrm{~Hz}$, and the modulation frequency peak $\left(f p_{m}\right)$ is located in this band with $\Delta f_{m}=0.02 \mathrm{~Hz}$. The respiratory frequency peak $\left(f p_{r}\right)$ is tracked in the band from 0.2 to $0.4 \mathrm{~Hz}$ with $\Delta f_{r}=0.2 \mathrm{~Hz}$. The individual powers of the two frequency bands $P_{m}$ and $P_{r}$ and their ratio $R=P_{m} / P_{r}$ constitute the three spectral parameters, which are investigated in this study, see Table I.

\section{EXPERIMENTAL PROTOCOL AND DATA ANALYSIS}

\section{A. Datasets}

Respiratory flow signals were recorded from 35 healthy volunteers ( 23 female, $27 \pm 7$ years, respiratory frequency $15.5 \pm$ 3.7 breaths $/ \mathrm{min}$ ) and $26 \mathrm{CHF}$ patients ( 7 female, $65 \pm 9$ years, $19.6 \pm 3.4$ breaths/min) at Santa Creu i Sant Pau Hospital, Barcelona, Spain. All subjects were studied according to a protocol approved by the local Ethics Committee. The respiratory flow signals were acquired using a pneumotachograph, consisting of a Datex-Ohmeda monitor with a Validyne Model MP451-871 Variable-Reluctance Transducer. The pneumotachograph was connected to a mask. The signals were recorded at $250-\mathrm{Hz}$ sampling rate and 12-bit resolution.

The same patient might present a mixture of breathing patterns, ranging from normal breathing (without cyclic modulation of ventilation) through mild PB to CSR patterns. The respiratory flow signals of $\mathrm{CHF}$ patients were acquired during a checkup. Prior to data acquisition, a few minutes of adaptation were allowed in order to make the subjects feel comfortable with the mask. The respiratory flow signals were acquired during $15 \mathrm{~min}$ in both CHF patients and healthy subjects. All subjects were seated and remained awake throughout acquisition.

The classification of the CHF patients (PB, nPB, and CSR) was developed by the medical doctors at the Santa Creu i Sant Pau Hospital, Barcelona, Spain. The CHF patients were classified into two groups: eight patients with PB patterns (1 female, $71 \pm 7$ years, $18.4 \pm 2.2$ breaths/min), and 18 patients with nPB pattern ( 5 female, $62 \pm 9$ years, $22.5 \pm 4.3$ breaths/min). Within the PB group, three patients were classified as CSR ( 1 female, $68 \pm 6$ years, $21.7 \pm 4.2$ breaths $/ \mathrm{min}$ ) and five patients as PB without apnea (no females, $73 \pm 8$ years, $23.0 \pm$ 4.7 breaths/min). Fig. 1 illustrates the different flow patterns observed in CSR, PB, nPB patients, and a healthy subject.

For the 61 subjects, the respiratory frequency was found to range from 0.2 to $0.4 \mathrm{~Hz}$, and the modulation frequency from 0.01 to $0.04 \mathrm{~Hz}$. These frequency ranges are in good agreement with those earlier reported by Pinna et al. [9]. 


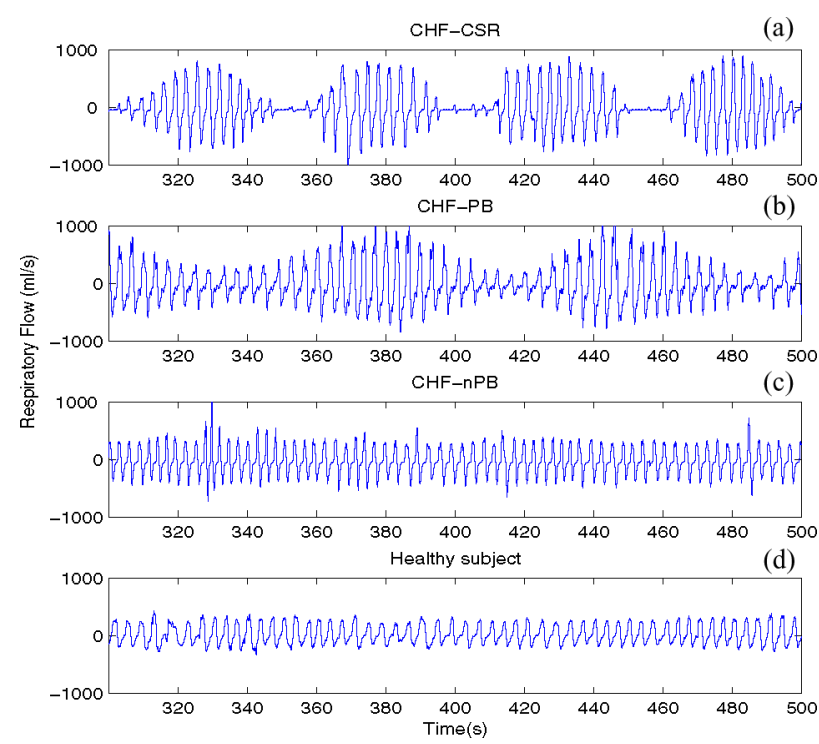

Fig. 1. Respiratory flowsignal exemplified for (a) a CSR patient, (b) a PB patient, (c) a nPB patient, and (d) a healthy subject.
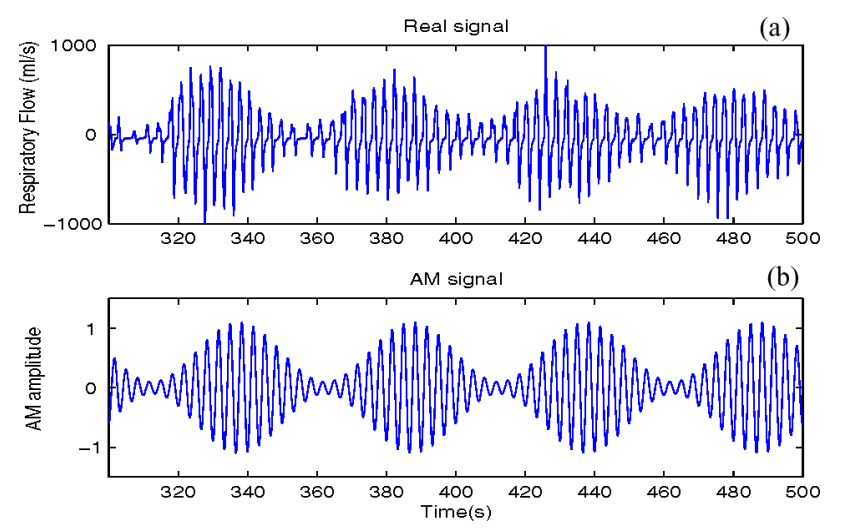

Fig. 2. (a) Respiratory flow signal acquired from a PB patient, and (b) a simulated AM signal with $0.3-\mathrm{Hz}$ carrier ("respiratory") frequency, $0.02-\mathrm{Hz}$ modulation frequency, and $\mu=0.8$.

The flow signals were decimated from 250 to $2 \mathrm{~Hz}$, using null-phase antialiasing filtering, to account for the fact that the frequencies of interest only ranges up to about $0.5 \mathrm{~Hz}$; oversampling is of particular importance to avoid when performing AR spectral analysis [30].

\section{B. Simulated Data}

The behavior of the respiratory flow signal in PB patients often resembles that of a signal subjected to amplitude modulation (AM). In order to illustrate certain properties of the method's performance, the following AM signal is studied

$$
x(n)=\left(1+\mu \cos \left(\omega_{m} n\right)\right) \cos \left(\omega_{c} n\right)
$$

where $f_{c}=\omega_{c} / 2 \pi$ is the carrier frequency, $f_{m}=\omega_{m} / 2 \pi$ is the modulation frequency, and $\mu \in[0,1]$ is the modulation index. Fig. 2 shows an example where the characteristics of the respiratory flow signal and the AM signal are quite similar.
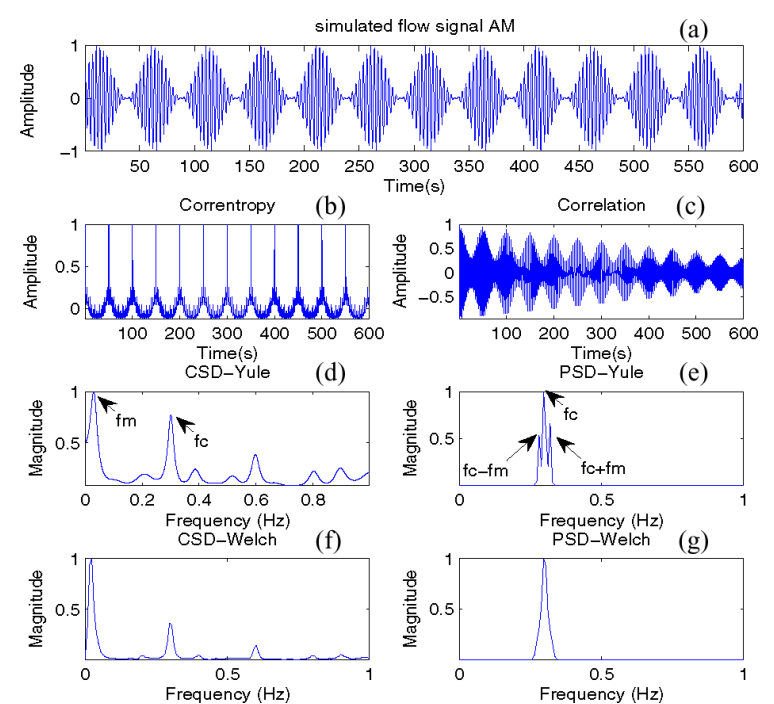

Fig. 3. (a) Simulated AM signal with $0.3-\mathrm{Hz}$ carrier frequency, 0.02-Hz modulation frequency, and $\mu=1$, (b) its correntropy function and (c) correlation function. The CSD obtained with (d) Yule-Walker's method and (f) Welch's method. The PSD obtained with (e) Yule-Walker's method and (g) Welch's method.

\section{Data Analysis}

The accuracy of the proposed parameters for pattern characterization is evaluated in terms of the following three classification problems: CHF patients with either $\mathrm{PB}$ or $\mathrm{nPB}, \mathrm{CHF}$ patients versus healthy subjects, and $\mathrm{nPB}$ patients versus healthy subjects. Using a linear classification procedure, a parameter selection process, being based on the leave-one-out crossvalidation, is employed in which only the single most discriminative parameter is considered.

\section{RESULTS}

The global AR model order was found to be 30 when the aforementioned selection procedure was applied. Applying the Ljung-Box test, the residuals of all 61 signals except two were found to be whitened by the global AR model. The sole design parameter of the method is $\sigma$, here determined by Silverman's rule in (6), and is investigated below for some deviating values. In order to make the figures comparable, the scales have been normalized to unity.

\section{A. Simulated Signals}

Fig. 3 illustrates the performance of the aforementioned methods in terms of power spectrum of the AM signal. From this figure, it is evident that the spectra based on either correntropy or correlation have very different characteristics, the main difference being the location of $f_{m}$. For the correntropy-based spectra, $f_{m}$ is found in the baseband and $f_{c}$ is accompanied with a number of harmonics. For the correlation-based spectra, the peaks are located at $f_{c}, f_{m}-f_{c}$, and $f_{m}+f_{c}$, respectively, as suggested by classical AM analysis. Note though that Welch's method is unable to resolve the two peaks at $f_{m}-f_{c}$ and $f_{m}+f_{c}$ [see Fig. 3(e)]; no harmonic pattern is present in the correlation-based spectra. Thus, correntropy-based spectra 

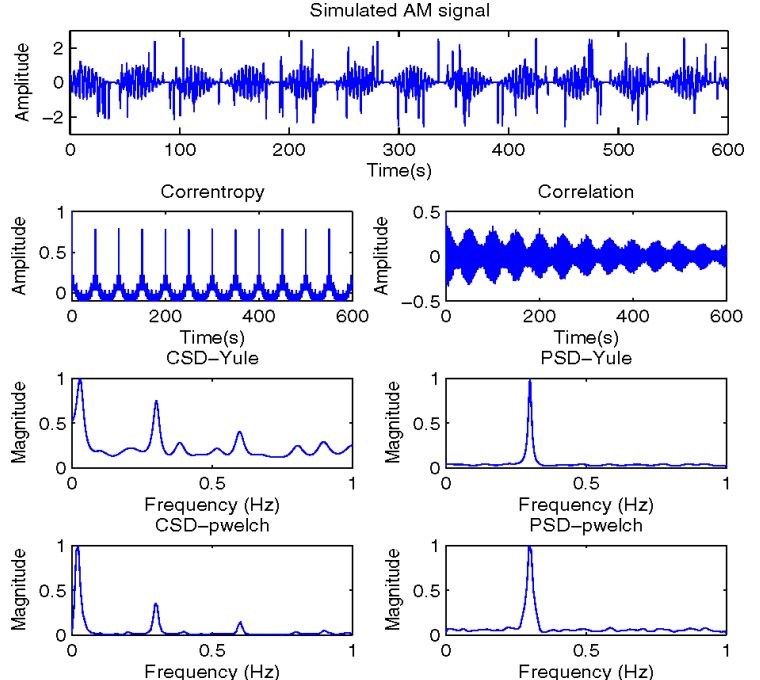

Fig. 4. (a) Simulated AM signal $(0.3-\mathrm{Hz}$ carrier frequency, $0.02-\mathrm{Hz}$ modulation frequency, and $\mu=1$ ) with some outliers randomly added, (b) its correntropy function and (c) correlation function. The CSD obtained with (d) Yule-Walker's method and (f) Welch's method. The PSD obtained with (e) Yule-Walker's method and (g) Welch's method.

has the advantage of exhibiting peaks at the positions of $f_{m}$ and $f_{c}$, which are easy to detect. On the other hand, the sideband peaks of the correlation-based spectra can be difficult to detect as they tend to smear with the respiratory peak at $f_{c}$.

Fig. 4 illustrates the robustness of the correntropy technique by adding randomly occurring impulsive noise to the AM signal analyzed in Fig. 3. It is noted that the modulation peaks present in Fig. 3(e) can no longer be discerned in Fig. 4(e).

\section{B. Real Signals}

Spectral analysis is also performed on signals from CHF patients (see Figs. 5-7) and a healthy subject (see Fig. 8) for the purpose of illustrating differences in respiratory patterns. In both types of spectra, the respiratory peak is clearly visible. The correntropy-based spectra of the healthy subject [see Fig. 8(d)] lacks a peak corresponding to the modulation frequency, unlike the spectra of $\mathrm{CHF}$ patients. The PB patients present much more prominent modulation peaks [see Figs. 5(d) and 6(d)] than does the nPB patient [see Fig. 6(d)]. It is evident from these examples that the modulation frequency peak is easily detected in the correntropy-based spectra, but not in the correlation-based spectra. In fact, the latter type of spectrum does not even lend itself to sideband peak detection.

\section{Sensitivity to $\sigma$}

The choice of $\sigma$ is illustrated for an AM signal and a flow signal using the CSD, see Fig. 9. The $\sigma$-value is chosen with reference to the optimal value $\sigma_{\text {opt }}$ given by Silverman's rule. For the AM signal, it is obvious that the power of the modulation peak decreases as $\sigma$ increases, i.e., $10 \sigma_{\text {opt }}$, whereas the power of the spurious harmonics increases as $\sigma$ decreases, i.e., $0.1 \sigma_{\text {opt }}$. When considering these three $\sigma$-values, the two peaks of interest are most easily detected for $\sigma_{\mathrm{opt}}$. For the flow signal, a similar
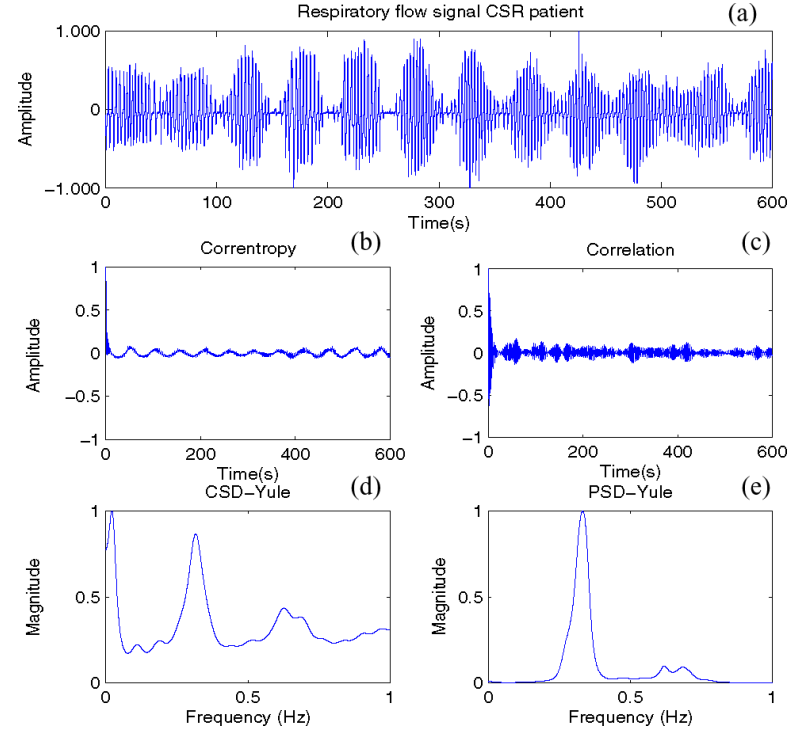

Fig. 5. (a) Respiratory flow signal of a CSR patient, (b) its correntropy function, and (c) correlation function. (d) CSD and (e) PSD, both obtained with Yule-Walker's method.
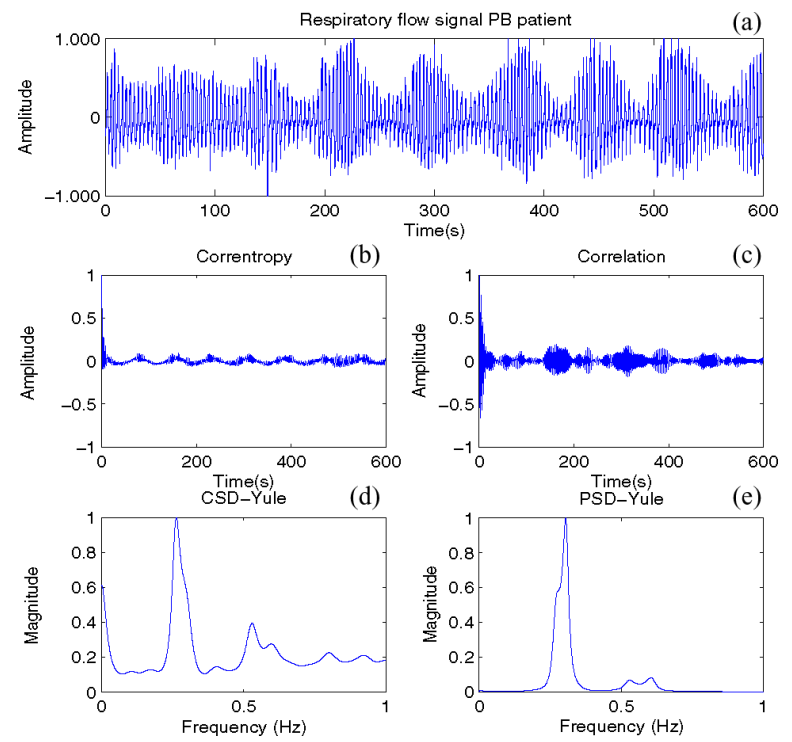

Fig. 6. (a) Respiratory flow signal of a CHF patient with $\mathrm{PB}$, (b) its correntropy function, and (c) correlation function. (d) CSD and (e) PSD, both obtained with Yule-Walker's method.

behavior is observed though the changes in spectral shape are less pronounced for different values of $\sigma$ than what is observed for the AM signal.

\section{Performance Evaluation}

Table II presents the mean and the standard deviation of the most relevant parameters, for each group of patients and healthy subjects. Table III shows the classification results obtained when only the most discriminative parameter was applied. The correntropy mean $\bar{V}$ provides the best results when classifying $\mathrm{CHF}$ patients versus healthy subjects and nPB patients versus healthy subjects, (95.2\% and $94.4 \%$, respectively). When classifying 

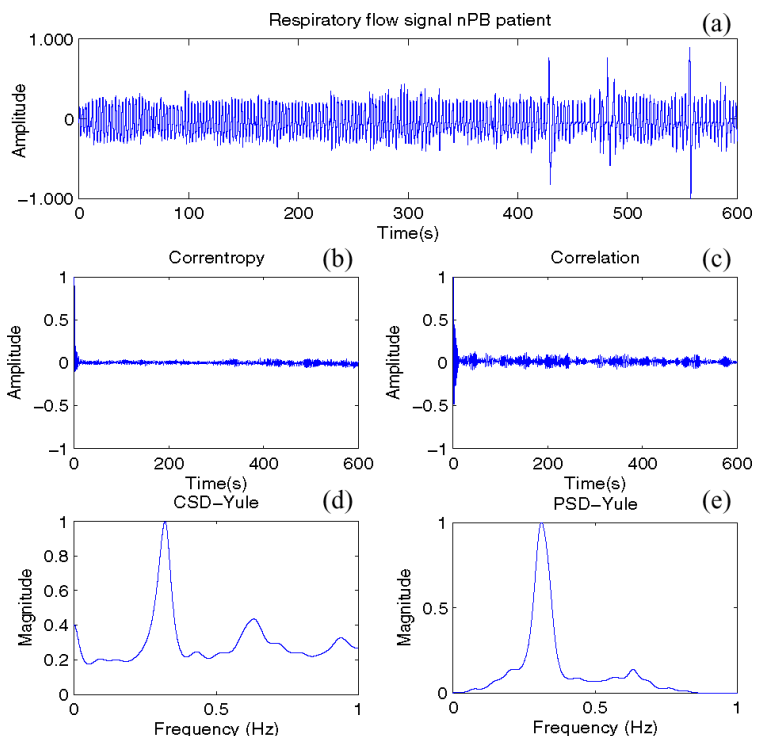

Fig. 7. (a) Respiratory flow signal of a CHF patient with $\mathrm{nPB}$, (b) its correntropy function, and (c) correlation function. (d) CSD and (e) PSD, both obtained with Yule-Walker's method.
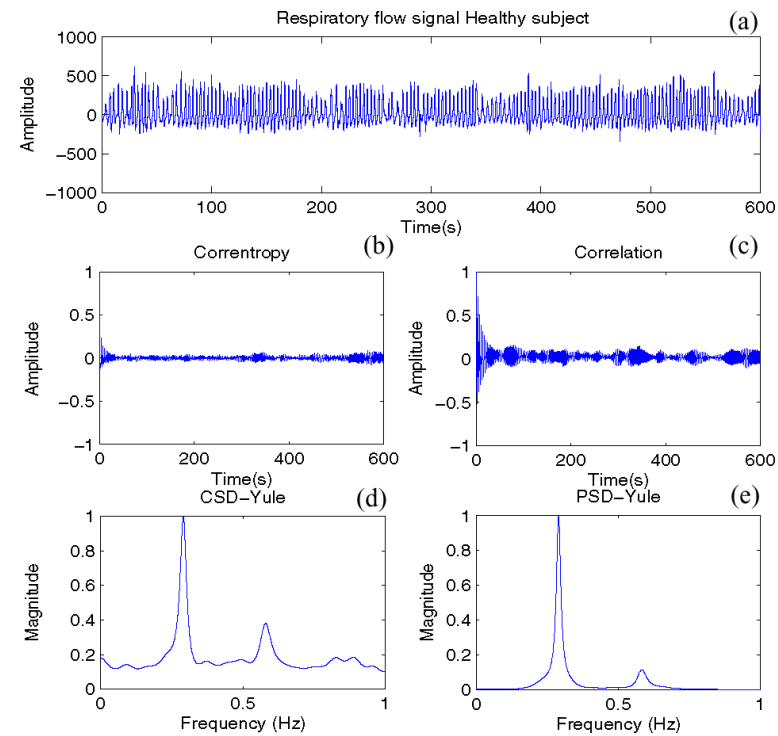

Fig. 8. (a) Respiratory flow signal of a healthy subject, (b) its correntropy function, and (c) correlation function. (d) CSD and (e) PSD, both obtained with Yule-Walker's method.

$\mathrm{PB}$ and $\mathrm{nPB}$ patients within the CHF group, only the spectral power-related parameters were found to produce good results, providing an accuracy of $88.9 \%$.

The sensitivity to different values of $\sigma$ is evaluated as before for $0.1 \sigma_{\mathrm{opt}}, \sigma_{\mathrm{opt}}$, and $10 \sigma_{\mathrm{opt}}$ with respect to the three classification tasks involving only the most discriminative parameter. Table IV presents the area under the receiver operating characteristic (ROC) for the three different classification tasks: it is obvious that the choice of $\sigma$ is not critical for the classification as the area under the ROC is essentially unchanged for the different values of $\sigma$.
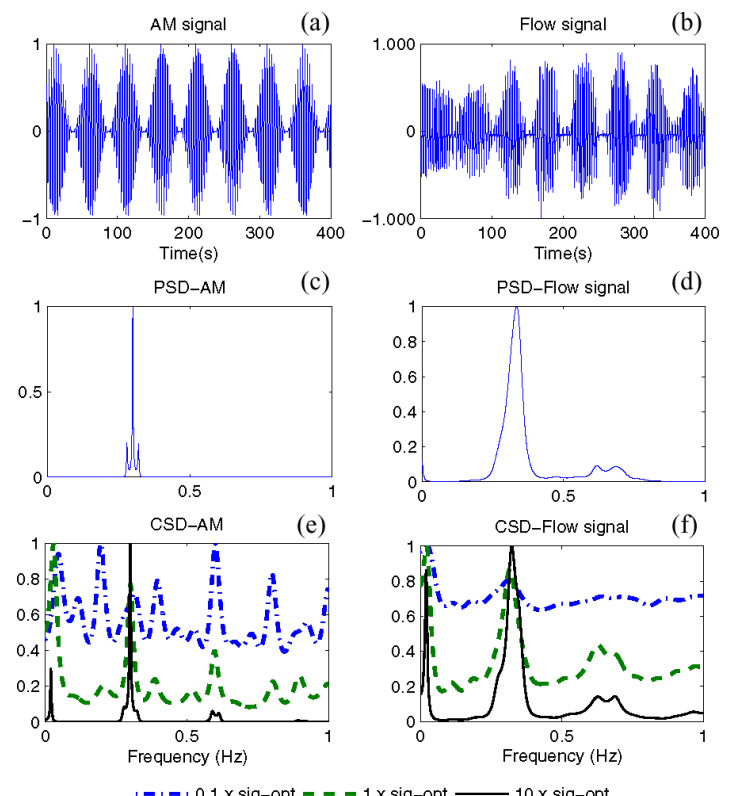

Fig. 9. (a) Simulated AM signal and (b) respiratory flow signal of a PB patient Their respective (c)-(d) PSDs and (e)-(f) CSDs evaluated for the kernel values $0.1 \sigma_{\mathrm{opt}}, \sigma_{\mathrm{opt}}$, and $10 \sigma_{\mathrm{opt}}$.

TABLE II

MEAN AND STANDARD DEVIATION OF $\bar{V}$ AND $R$ FOR EACH GROUP OF SUBJECTS*

\begin{tabular}{lcc}
\hline & $\bar{V}$ & $R$ \\
\hline Healthy & $0.12 \pm 0.05$ & $8.4 \pm 8.4$ \\
CHF & $0.04 \pm 0.02$ & $9.2 \pm 4.9$ \\
PB & $0.03 \pm 0.01$ & $14.2 \pm 5.8$ \\
nPB & $0.04 \pm 0.02$ & $7.1 \pm 2.4$ \\
\hline
\end{tabular}

TABLE III

SENSITIVITY (SN), SPECIFICITY (SP), AND ACCURACY (ACC) OBTAINED WITH THE BEST PARAMETER FOR EACH CLASSIFICATION USING LEAVE-ONE-OUT CROSS-VALIDATION

\begin{tabular}{lcccc}
\hline Classification & Parameter & Sn & Sp & Acc \\
\hline CHF vs. Healthy & $\bar{V}$ & $96.3 \%$ & $94.3 \%$ & $95.2 \%$ \\
nPB-CHF vs. Healthy & $\bar{V}$ & $94.7 \%$ & $94.3 \%$ & $94.4 \%$ \\
PB vs. nPB (CHF) & $R$ & $75.0 \%$ & $94.7 \%$ & $88.9 \%$ \\
\hline
\end{tabular}

TABLE IV

Sensitivity of THE Kernel PARAmeter $\sigma$ IN TERms of AREA Under the ROC OBTAINED FOR THE BEST PARAMETER FOR EACH CLASSIFICATION

\begin{tabular}{lcccc}
\hline Area under the ROC & & $0.1 \sigma_{o p t}$ & $\sigma_{o p t}$ & $10 \sigma_{o p t}$ \\
\hline CHF vs. Healthy & $(\bar{V})$ & 0.98 & 0.98 & 0.98 \\
nPB-CHF vs. Healthy & $(\bar{V})$ & 0.97 & 0.97 & 0.98 \\
PB vs. nPB (CHF) & $(R)$ & 0.70 & 0.88 & 0.90 \\
\hline
\end{tabular}

\section{DISCUSSION}

This paper investigates the use of correntropy for spectral characterization of respiratory patterns in CHF patients. In contrast to the conventional second-order correlation function, the correntropy function accounts for higher order moments and is robust to outliers. In this study, the former property translates to 
the fact that the respiratory and modulation frequencies appear at their actual locations along the frequency axis in the CSD; see the Appendix for an explanation of this property. Hence, the correntropy circumvents problems that may arise when identifying the modulation frequency peak directly from the conventional PSD due to insufficient spectral resolution; this problem is illustrated for the simulated AM signal in Fig. 3. In the same sense, the use of correntropy eliminates the demodulation step, which was an integral part of our previous studies [19], [20], being introduced so that respiratory periodicity could be detected from the envelope of the flow signal with time-frequency analysis. In those two studies, various parameters were derived from the time-frequency representation and employed for classifying respiratory patterns in CHF patients. Using a three-parameter procedure, which involved the AR coefficients, an accuracy of $84.6 \%$ was achieved when classifying PB and nPB patients [20]. Obviously, that result is inferior to the one achieved here with an accuracy of $88.9 \%$, see Table III. While this improvement is perhaps not dramatic it is still of importance as the present classification is based on only one parameter instead of three. The inferior result of the previous study was primarily due to difficulties in identifying the modulation frequency.

Another attractive property of the correntropy function is its robustness against outliers, explained by the fact that the inner product is computed via the Gaussian kernel in the parameter space, which tends to zero when outliers occur. Such robustness is essential in the analysis of respiratory signals since they are frequently corrupted with noise and spike artifacts. For the present study, this property has implied that the preprocessing required for outlier rejection in our previous studies, [19], [20], could be completely eliminated without impairing classification performance. Accordingly, the present method is considerably more efficient from a computational viewpoint than our previous methods.

The choice of $\sigma$ was found to be uncritical as rather large changes in value did not alter performance much. Using Silverman's rule, the performance in terms of sensitivity and specificity remained essentially the same even if the value was ten times larger or ten times smaller compared to the value given by the rule, see Table IV. A further increase of $\sigma$ causes however the importance of higher-order moments to decay much faster so that the correntropy approaches the second-order correlation, whereas a further decrease in $\sigma$ leads to meaningless spectral estimation.

It should be noted that the proposed method is very simple in structure because $\sigma$ constitutes the only design parameter. The model order, which by some would be treated as a design parameter [31], [30], was held fixed for all subjects once estimated from the dataset. The chosen model order of 30 turns out to be less critical as classification performance was virtually identical when orders between 25 and 35 were used instead.

The main goal of this study has been to identify CHF patients' condition noninvasively by characterizing and classifying respiratory flow patterns that derive from patients with $\mathrm{PB}$ and $\mathrm{nPB}$. The best results were, in general, achieved with CSD-related parameters characterizing the power in $\Delta f_{m}$ and $\Delta f_{r}$, i.e., $P_{m}$ and $R$. These parameters achieved the best results in all classifica- tions performed with CHF patients consisting of CSR patients, PB patients without apnea, and nPB patients. It should be noted that all patients, i.e., both $\mathrm{PB}$ and $\mathrm{nPB}$, exhibit various degrees of periodicity depending on their condition, whereas the healthy subjects have no pronounced periodicity.

Since patients with PB patterns exhibit a larger modulation peak in proportion to the respiratory peak than do nPB patients, the ratio between the power in the modulation band and respiration band is a suitable parameter for classifying $\mathrm{PB}$ and $\mathrm{nPB}$ patients. The promising classification results are explained by the fact that the CSD provides a more clear-cut representation of the modulation peak than does the PSD. In general, CHF patients present an increased amplitude on ventilation and therefore on their respiratory flow signal. Since the correntropy is based on the distance between samples, the correntropy mean turns out to be higher in healthy subjects than in the CHF patients. It provides excellent classification results in the classification of $\mathrm{CHF}$ and healthy subjects, as well as of nPB patients and healthy subjects, CHF verus healthy $95.2 \%$, and $\mathrm{nPB}$ versus healthy $94.4 \%$ ).

The main goal of most studies on breathing patterns in CHF patients differ from the present one as other studies are focused on the prediction of mortality, e.g., by exploring the properties of heart rate variability with linear [32], [33] and nonlinear techniques [34]. Consequently, it is difficult to compare the present results with those obtained in other studies.

The small size of the dataset is a limitation of the present study, and therefore the significance of the results, though being promising, needs to be further established on a larger set. As a consequence of the small size, it was decided to only investigate the performance of single-parameter linear classification.

\section{CONCLUSION}

In this study, the correntropy-based spectral density is found to be particularly well suited for the characterization of modulated breathing patterns. Three different spectral parameters are defined, which characterize the power in the respiratory and modulation frequency bands and their proportion. The spectral estimation method is simple as very few design parameter values need to be set. However, research is needed to better understand how the eigenstructure of the correntropy matrix influences the estimation of AR parameters. Simulations with an AM signal demonstrate that the correntropy approach comes with much better spectral resolution than does the conventional techniques. Though this study is preliminary in nature, the classification results on a clinical dataset, containing respiratory flow signals from CHF patients and healthy subjects, are promising as they surpass earlier results obtained for a demodulation-based method.

\section{APPENDIX}

This appendix offers an explanation to why the correntropy makes the respiratory and modulation frequency peaks more discernible without requiring demodulation. The starting point is the AM signal,

$$
x(n)=(1+\mu M(n)) C(n)
$$



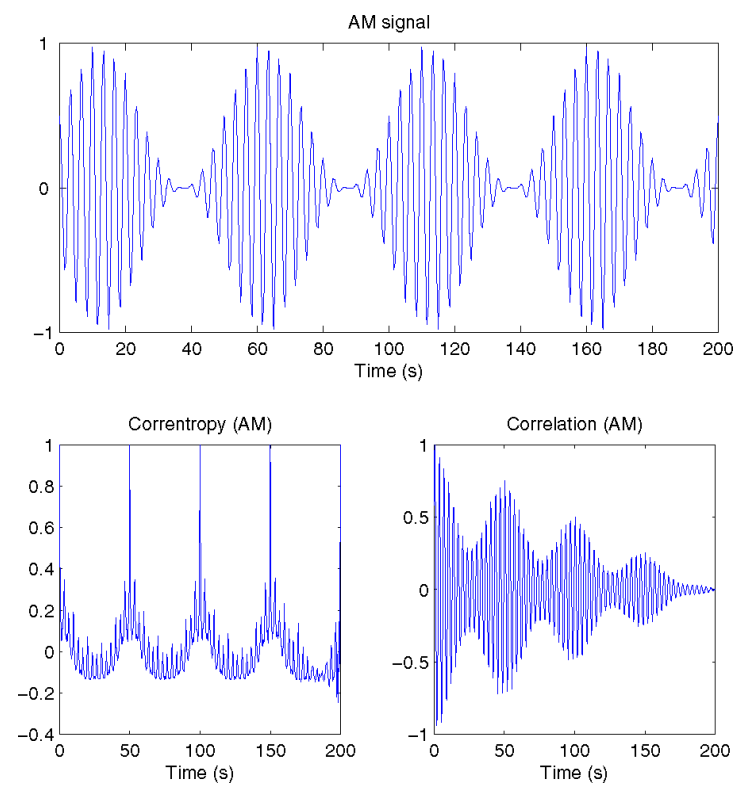

Fig. 10. (a) AM signal and (b) its correntropy function, using $\sigma$ given by Silverman's rule, and (c) its correlation function.

where $M(n)=\cos \left(\omega_{m} n\right)$ and $C(n)=\cos \left(\omega_{c} n\right)$. The corresponding correlation function is given by

$$
R_{x}(n)=\left(1+\mu^{2} R_{M}(n)\right) \cos \left(\omega_{c} n\right)
$$

where $R_{M}(n)$ denotes the correlation function of $M(n)$. Thus, $R_{x}(n)$ is proportional to $R_{M}(n)$, being modulated by $\omega_{c}$.

On the other hand, it can be shown that the correntropy function of $x(n)$ is proportional to both $V_{M}(n)$ and $V_{C}(n)$. For a Gaussian kernel, $V_{M}(n)$ reaches its maximum only when $M(n)=M(n+m)$. When this occurs, we have that $\kappa\left(x_{n}-x_{n-m}\right)=1 / \sqrt{2 \pi} \sigma$, and $M(n)=M(n+$ $m) \rightarrow \cos \left(\omega_{m} n\right)=\cos \left(\omega_{m}(n+m)\right), \Leftrightarrow m \omega_{m} n=2 \pi k, k=$ $1,2, \ldots$ The maximum values are obtained when $m=m_{m}=$ $2 \pi k / \omega_{m}=T_{m} F_{s} k$, where $F_{s}$ is the sampling rate and $T_{m}$ is the modulation period. The correntropy $V_{M}(n)$ reaches its maximum for each $m_{m}$ and decreases rapidly when the difference $M(n)-M(n+m)$ increases. For $V_{C}(n)$, the maximum values are reached when $m=m_{c}=2 \pi k / \omega_{c}=T_{c} F_{s} k$, where $T_{c}$ is the carrier period.

The correntropy function $V(n)$ reaches its maximum when $m$ is a multiple of both $T_{m}$ and $T_{c}$, whereas the relative maximum values are reached when $m=m_{c}$. As $\omega_{m} \leq \omega_{c} \rightarrow T_{m} \geq T_{c} \rightarrow$ $m_{m} \geq m_{c}$. Thus, the maximum value is given by the modulation signal $\left(m_{m}\right)$.

Only when both periods are multiples, i.e., $T_{m}=k T_{c}$ is the maximum value $1 / \sqrt{2 \pi} \sigma$ reached, otherwise this value is lower. The maximum value appears in each modulation period, explaining why the modulation frequency peak is sometimes larger than the respiratory frequency peak. Thus, it can be stated that correntropy enhances the modulation peak. Fig. 10 demonstrates the property that $R_{x}(n) \propto R_{M}(n)$ is modulated by $C(n)$, whereas $V(n) \propto \gamma V_{M}(n)+\beta V_{C}(n)$, where $\gamma$ and $\beta$ are positive constants.

\section{ACKNOWLEDGMENT}

The authors would like to thank Dr. S. Benito and Dr. A. Bayés-Genis and their colleagues at Santa Creu i Sant Pau Hospital, Barcelona, Spain, for fruitful collaboration concerning the signal database acquisition.

\section{REFERENCES}

[1] S. Neubauer, "The failing heart-an engine out of fuel," New Engl. J. Med., vol. 356, no. 11, pp. 1140-1151, 2007.

[2] M. Dayer and M. R. Cowie, "Heart failure: Diagnosis and healthcare burden," Clin. Med. (Londond, England), vol. 4, no. 1, pp. 13-18, 2004.

[3] G. Lorenzi-Filho, P. R. Genta, A. C. Figueiredo, and D. Inoue, "CheyneStokes respiration in patients with congestive heart failure: Causes and consequences," Clinics (Sao Paulo, Brazil), vol. 60, no. 4, pp. 333-344, 2005.

[4] D. P. Francis, K. Willson, L. C. Davies, A. J. Coats, and M. Piepoli, "Quantitative general theory for periodic breathing in chronic heart failure and its clinical implications," Circulation, vol. 102, no. 18, pp. 2214-2221, 2000.

[5] G. D. Pinna, R. Maestri, A. Mortara, P. Johnson, T. Witkowski, P. Ponikowski, D. Andrews, S. Capomolla, M. T. La Rovere, and P. Sleight, "Nocturnal periodic breathing is an independent predictor of cardiac death and multiple hospital admissions in heart failure," Proc. Comput. Cardiol., vol. 33, pp. 837-840, 2006.

[6] M. Guazzi, R. Raimondo, M. Vicenzi, R. Arena, C. Proserpio, S. Sarzi Braga, and R. Pedretti, "Exercise oscillatory ventilation may predict sudden cardiac death in heart failure patients," J. Amer. Coll. Cardiol., vol. 50, no. 4, pp. 299-308, 2007.

[7] R. Poletti, C. Passino, A. Giannoni, L. Zyw, C. Prontera, F. Bramanti, A. Clerico, M. Piepoli, and M. Emdin, "Risk factors and prognostic value of daytime cheyne-stokes respiration in chronic heart failure patients," Int. J. Cardiol., vol. 137, no. 1, pp. 47-53, 2008.

[8] U. Corra, M. Pistono, A. Mezzani, A. Braghiroli, A. Giordano, P. Lanfranchi, E. Bosimini, M. Gnemmi, and P. Giannuzzi, "Sleep and exertional periodic breathing in chronic heart failure: Prognostic importance and interdependence," Circulation, vol. 113, no. 1, pp. 44-50, 2006.

[9] G. D. Pinna, R. Maestri, A. Mortara, M. T. La Rovere, F. Fanfulla, and P. Sleight, "Periodic breathing in heart failure patients: testing the hypothesis of instability of the chemoreflex loop," J. Appl. Physiol., vol. 89, no. 6, pp. 2147-2157, 2000.

[10] P. Ponikowski, S. D. Anker, T. P. Chua, D. Francis, W. Banasiak, P. A Poole-Wilson, A. J. Coats, and M. Piepoli, "Oscillatory breathing patterns during wakefulness in patients with chronic heart failure: Clinical implications and role of augmented peripheral chemosensitivity," Circulation, vol. 100, no. 24, pp. 2418-2424, 1999.

[11] J. P. Ribeiro, "Periodic breathing in heart failure: Bridging the gap between the sleep laboratory and the exercise laboratory," Circulation, vol. 113, no. 1, pp. 9-10, 2006.

[12] A. Vazir, M. Dayer, P. C. Hastings, H. F. McIntyre, M. Y. Henein, P. A. Poole-Wilson, M. R. Cowie, M. J. Morrell, and A. K. Simonds, "Can heart rate variation rule out sleep-disordered breathing in heart failure?" Eur. Resp. J., vol. 27, no. 3, pp. 571-577, 2006.

[13] P. J. Hanly and N. S. Zuberi-Khokhar, "Increased mortality associated with Cheyne-Stokes respiration in patients with congestive heart failure," Amer. J Resp. Crit. Care Med., vol. 153, no. 1, pp. 272-276, 1996.

[14] S. Javaheri, T. J. Parker, J. D. Liming, W. S. Corbett, H. Nishiyama, L. Wexler, and G. A. Roselle, "Sleep apnea in 81 ambulatory male patients with stable heart failure. Types and their prevalences, consequences, and presentations," Circulation, vol. 97, no. 21, pp. 2154-2159, 1998.

[15] Y. Kaneko, J. S. Floras, K. Usui, J. Plante, R. Tkacova, T. Kubo, S. Ando and T. D. Bradley, "Cardiovascular effects of continuous positive airway pressure in patients with heart failure and obstructive sleep apnea," New Engl. J. Med., vol. 348, no. 13, pp. 1233-1241, 2003.

[16] D. R. Mansfield, N. C. Gollogly, D. M. Kaye, M. Richardson, P. Bergin, and M. T. Naughton, "Controlled trial of continuous positive airway pressure in obstructive sleep apnea and heart failure," Amer. J. Resp. Crit. Care Med., vol. 169, no. 3, pp. 361-366, 2004.

[17] A. Mortara, P. Sleight, G. D. Pinna, R. Maestri, A. Prpa, M. T. La Rovere, F. Cobelli, and L. Tavazzi, "Abnormal awake respiratory patterns are common in chronic heart failure and may prevent evaluation of autonomic tone by measures of heart rate variability," Circulation, vol. 96, no. 1, pp. 246-252, 1997. 
[18] U. Corra, A. Giordano, E. Bosimini, A. Mezzani, M. Piepoli, A. J. Coats, and P. Giannuzzi, "Oscillatory ventilation during exercise in patients with chronic heart failure: Clinical correlates and prognostic implications," Chest, vol. 121, no. 5, pp. 1572-1580, 2002.

[19] A. Garde, B. Giraldo, R. Jané, I. Diaz, S. Herrera, S. Benito, M. Domingo, and A. Bayes-Genis, "Analysis of respiratory flow signals in chronic heart failure patients with periodic breathing," in Proc. IEEE Conf. Eng. Med. Biol., 2007, pp. 307-310.

[20] A. Garde, B. F. Giraldo, R. Jané, I. Diaz, S. Herrera, S. Benito, M. Domingo, and A. Bayes-Genis, "Characterization of periodic and nonperiodic breathing pattern in chronic heart failure patients," in Proc. IEEE Conf. Eng. Med. Biol., 2008, pp. 3227-3230.

[21] I. Santamaria, P. P. Pokharel, and J. C. Principe, "Generalized correlation function: definition, properties, and application to blind equalization," IEEE Trans. Signal Process., vol. 54, no. 6, pp. 2187-2197, Jun. 2006.

[22] W. Liu, P. P. Pokharel, and J. C. Principe, "Correntropy: Properties and applications in non-Gaussian signal processing," IEEE Trans. Signal Process., vol. 55, no. 11, pp. 5286-5298, Nov. 2007.

[23] J. W. Xu and J. C. Principe, "A pitch detector based on a generalized correlation function," IEEE Trans. Audio, Speech, Lang. Process., vol. 16, no. 8, pp. 1420-1432, Aug. 2008.

[24] K.-H. Jeong, W. Liu, S. Han, E. Hasanbelliu, and J. C. Principe, "The correntropy mace filter," Pattern Recogn., vol. 42, no. 5, pp. 871-885, 2009.

[25] V. N. Vapnik, Statistical Learning Theory. New York: Wiley, 1998.

[26] M. G. Genton, "Classes of kernels for machine learning: A statistics perspective," J. Mach. Learn. Res., vol. 2, pp. 299-312, 2002.

[27] Y. Rosen and B. Porat, "The second-order moments of sample covariances for time series with missing observations," IEEE Trans. Info. Theory, vol. 35, no. 2, pp. 334-341, Mar. 1989.

[28] J. Rissanen, "Modeling by shortest data description," Automatica, vol. 14, pp. 465-471, 1978.

[29] C. W. Therrien, Discrete Random Signals and Statistical Signal Processing. Englewood Cliffs: NJ: Prentice-Hall, 1992.

[30] L. Sörnmo and P. Laguna, Bioelectrical Signal Processing in Cardiac and Neurological Applications. Amsterdam, The Netherlands/New York: Elsevier/Academic, 2005.

[31] G. E. Birch, P. D. Lawrence, J. C. Lind, and R. D. Hare, "Application of prewhitening to AR spectral estimation of EEG," IEEE Trans. Biomed. Eng., vol. 35, no. 8, pp. 640-645, Aug. 1988.

[32] M. Galinier, A. Pathak, J. Fourcade, C. Androdias, D. Curnier, S. Vamous, S. Boveda, P. Massabau, M. Fauvel, J. M. Senard, and P. Bounhoure, "Depressed low frequency power of heart rate variability as an independent predictor of sudden death in chronic heart failure," Eur. Heart J., vol. 21, pp. $475-482,2000$.

[33] M. T. La Rovere, G. D. Pinna, R. Maestri, A. Mortara, S. Capomolla, O. Febo, R. Ferrari, M. Franchini, M. Gnemmi, C. Opasich, P. G. Riccardi, and E. Traversi, "Short-term heart rate variability predicts sudden cardiac death in chronic heart failure patients," Circulation, vol. 107, pp. 565-570, 2003.

[34] N. M. Arzeno, M. T. Kearney, D. L. Eckberg, J. Nolan, and C. S. Poon, "Heart rate chaos as a mortality predictor in mild to moderate heart failure," in Proc. IEEE Conf. Eng. Med. Biol., 2007, pp. 5051-5054.

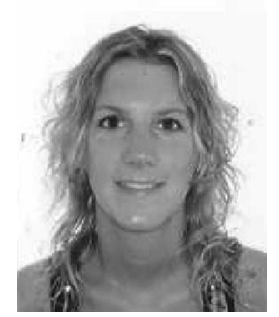

Ainara Garde (S'09) was born in Irunea, Nafarroa, in 1979. She received the M.Sc. degree in telecommunication engineering from the Public University of Pamplona, Pamplona, Spain, in 2004. She is currently working toward the Ph.D degree from the Departament d'Enginyeria de Sistemes, Automàtica i Informàtica Industrial, Universitat Politècnica de Catalunya, and the Institut de Bioenginyeria de Catalunya in Barcelona, Spain.

She is also with the Centro de Investigación Biomédica en Red de Bioingeniería, Biomateriales y Nanomedicina, Barcelona, Spain. In 2008, she was a Visiting Researcher at the Department of Medical Engineering, University of Applied Sciences, Jena, Germany, and at the Signal Processing Group, Department of Electrical and Information Technology and Centre of Integrative Electrocardiology, Lund University, Sweden.Her current research interests include signal processing and modeling of biomedical signals to characterize respiratory pattern and applications of machine learning methods to solve biomedical problems.

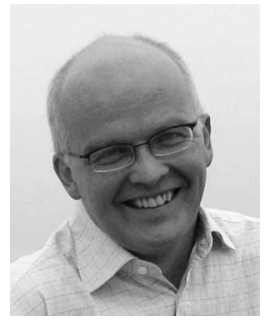

Leif Sörnmo (S'80-M'85-SM'02) received the M.Sc. and Ph.D. degrees in electrical engineering from Lund University, Lund, Sweden, in 1978 and 1984, respectively.

From 1983 to 1995 , he was a Researcher at the Department of Clinical Physiology, Lund University, where he was involved with problems in ECG signal processing. Since 1990, he has been with the Signal Processing Group, Department of Electrical and Information Technology, Lund University, where he is currently a Professor of biomedical signal processing. He is the author or coauthor of the book Bioelectrical Signal Processing in Cardiac and Neurological Applications (Elsevier, 2005). His main research interests include statistical signal processing and modeling of biomedical signals, methods for atrial fibrillation analysis, multimodal signal processing in hemodialysis, detection of otoacoustic emissions, and power-efficient signal processing in implantable devices.

Prof. Sörnmo is an Associate Editor of the IEEE TRANSACTIONS ON BIOMEDICAL ENGINEERING and the Journal of Electrocardiology, and on the editorial board of Medical and Biological Engineering and Computing; and has been an Associate Editor of Computers in Biomedical Research (1997-2000).

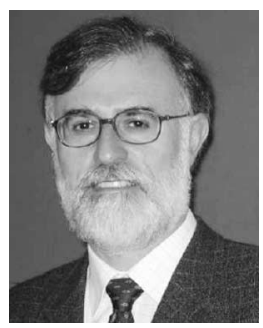

Raimon Jané (M'91) received the Ph.D. degree from the Universitat Politècnica de Catalunya (UPC), Barcelona, Spain, in 1989.

$\mathrm{He}$ is currently the Director of Research at the Departament d'Enginyeria de Sistemes, Automàtica i Informàtica Industrial, UPC, and a Scientific Group Leader of the Biomedical Signal Processing and Interpretation Group, Institut de Bioenginyeria de Catalunya, Barcelona, Spain. Since 2008, he has been the Principal Investigator of the Biomedical Signals and Systems Group, Centro de Investigación Biomédica en Red de Bioingeniería, Biomateriales y Nanomedicina, Barcelona, Spain. He is currently also a Professor of the Master's and Coordinator of the $\mathrm{Ph} . \mathrm{D}$. programmes on biomedical engineering. His research interests include multimodal and multiscale biomedical signal processing in cardiorespiratory and brain diseases.

Prof. Jané is a Vice-President of the Spanish Society of Biomedical Engineering and the International Advisory Board of Physiological Measurement. He was the recipient of the Barcelona City Prize, of the Barcelona Council, in the area of technology research in 2005.

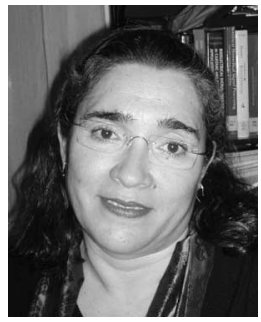

Beatriz F. Giraldo (M'00) received the M.Sc. degree in electrical engineering from the Technical University of Pereira, Pereira, Colombia, in 1983, and the M.Sc. degree in bioengineering, and the Ph.D. degree in industrial engineering in the Program of Biomedical Engineering from the Universitat Politècnica de Catalunya (UPC), Barcelona, Spain, in 1990 and 1996, respectively.

She is currently a "Colaborador Doctor" Professor at the Departament d'Enginyeria de Sistemes, Automàtica i Informàtica Industrial, UPC, a member of the Biomedical Signal Processing and Interpretation Group, Institut de Bioenginyeria de Catalunya, Barcelona, Spain, and a Researcher at the Biomedical Signals and Systems Group, Centro de Investigación Biomédica en Red en de Bioingeniería, Biomateriales y Nanomedicina, Barcelona. Since 2006, she has been the Director of the Bioengineering Program from the Fundació UPC. Her main research interests include biomedical signal processing and statistical analysis of cardiac, respiratory, and cardiorespiratory signals. 